\title{
A Method For Prioritizing Qualitative SCENARIOS IN EVALUATING ENTERPRISE ARCHITECTURE USING NON-DOMINATED SORTING GENETIC ALgORITHM II
}

\author{
Marzieh Eskandari and Akbar Nabiollahi \\ Faculty of Computer Engineering, Islamic Azad University, Najafabad Branch, \\ Najafabad, Iran
}

\begin{abstract}
In the field of enterprise architecture (EA), qualitative scenarios are used to understand the qualitative characteristics better. In order to reduce the implementation cost, scenarios are prioritized to be able to focus on the higher priority and more important scenarios. There are different methods to evaluate enterprise architecture including architecture Trade-off Analysis Method (ATAM).Prioritizing qualitative scenarios is one of the main phases of this method. Since none of the recent studies meet the prioritizing qualitative scenarios requirements, considering proper prioritizing criteria and reaching an appropriate speed priority, non-dominated sorting genetic algorithms is used in this study (NSGA-II). In addition to previous research standards more criteria were considered in the proposed algorithm, these sets of structures together as gene and in the form of cell array constitute chromosome. The proposed algorithm is evaluated in two case studies in the field of enterprise architecture and architecture software. The results showed the accuracy and the more appropriate speed comparing to the previous works including genetic algorithms.
\end{abstract}

\section{KEYWORDS}

enterprise architecture evaluation, qualitative scenarios prioritizing, NSGA-II algorithm

\section{INTRODUCTION}

Enterprise Architecture is a framework for the development and management of the organization's information technology resources to achieve organizational goals [1]. Architecture at the level of an organization is called enterprise architecture, the most important feature of which is providing a holistic view of the organization[2].In fact, architecture includes organizing a system consisting of a set of elements, the relationships of these elements with each other, with the environment as well as the guiding principles of designing and system development [3]. One of the most important achievements of enterprise architecture activity is enabling organizations to assess the currentsituation and also to identify, analyze, evaluate and select appropriate architecture solutions for organizations or in other words enterprise architecture evaluation. Recently different methods are proposed for evaluating enterprise architecture, each one having its own features and characteristics and in a way helping organizations' decision-making [2]. Enterprise architecture methods are conducted in different phases and stages. The areas of evaluation focus on detecting and diagnosing is whether the proposed architecture has the organization's desired qualitative characteristics. In the field of software architecture evaluation different methods are used which are based on qualitative scenarios. In this paper ATAM(Architecture Trade-off Analysis Method)method for Is used to evaluate enterprise 
architecture [4]. One of the main phases of this method is prioritizing qualitative scenarios. Among different evaluation methods, ATAMmethod is better for evaluation because of its tools, strategies, application scope and the existence of a very good applicable specimen [5].

As mentioned, due to the high cost of qualitative scenarios implementation scenarios are prioritizing so that we focus on the ones with the higher priority that are more important. Therefore, the qualitative scenarios prioritizing issue is a matter of decision-making affected by many different criteria [6]. Since none of the recent studies meets the prioritizing qualitative scenarios requirements, considering proper prioritizing criteria and reaching an appropriate speed priority, non-dominated sorting genetic algorithms is used in this study (NSGA-II), which considered more criteria for prioritizing scenarios and has a better speed comparing to previous methods like gene.

The proposed algorithm is evaluated in two case studies in the field of enterprise architecture and architecture software. In the first case study in the area of enterprise architecture the accuracy of the proposed algorithm is approved. The second case study shows that its speed is better than the speed of previous methods; since there is no repetitive scenario in the qualitative scenarios prioritizing, the exactness of this algorithm is better than the previous algorithms including genetic algorithm.

The structure of this article includes several parts: second part is the previous works on the enterprise architecture evaluation, the third part contains NSGA - II algorithm, the forth part focus on the clarification of NSGA - II algorithm, the algorithm is evaluated in the fifth part and finally the results are presented in the sixth section.

\section{LITERATURE REVIEW}

Several studies have been done in the area of enterprise architecture evaluation including the ones presented below. The method proposed by Schekkerman [7], this method is also known as enterprise architecture Scorecard. The mentioned method expresses a methodological line in order to evaluate the quality of the activities and enterprise architecture results. This method uses the developed architecture frameworks, but there is still insufficient accuracy in selecting qualitative characteristics [5]. The second method is the method provided by Management and Budget Office [8], the aim of this procedure is to help organizations understand and improve the current state of architecture. This method evaluates all aspects and criteria of enterprise architecture but there's not enough exactness in choosing the qualitative characteristics [8]. Methods provided by a group of researchers from the University of KTH in Sweden [9], [10], [11], [12], [13], [14 ] are next. These methods are different methods, which principles and concepts are different in some cases, but in this section, we have considered them as a group. This method supports organizational decisions but does not have enough accuracy in determining indexes [2]. Another method have also presented by Mr. Khayyami [5], in a part of his doctoral thesis, he determined the good qualitative characteristics of an enterprise architecture and proposed a clear and simple way to evaluate these characteristics. In this method, architecture models and information are analyzed but is based on expert knowledge that determines the value of criteria according to architecture models and information [5]. Ms. Razavi's method is the fifth one [2]. She presented a method in her PhD thesis that has two phases of determining the architecture qualitative characteristics and presenting a new framework for evaluating enterprise architecture.

In addition to introducing a new structure for qualitative scenarios, enterprise architecture is evaluated based on fuzzy logic in Razavi's method. Her method reduces human errors but it still does not have enough exactness in the prioritizing qualitative scenarios. She also presented an 
article for analyzingenterprise architecture entitled" An Approach towards Enterprise Architecture Analysis using Analytical Hierachy Process (AHP) and Fuzzy AHP ". The last work here is Mr. Karimi's method using genetic algorithm to prioritize qualitative scenarios [16], [17]. Comparing to previous methods, his considered more prioritizing criteria but it has several basic problems including lack of certainty in shareholders' views, not computing the accuracy [16] repetitive prioritizing of scenarios and low speed. There are a large number of scenarios in enterprise architecturedue to their wide range, the number of shareholders and their prioritizing criteria [18]. The main problem in most of these methods is that we need high speed for processing all these criteria and architecture analysis based on them but none of the introduced procedures find any solution for it.

\section{NSGA-II Proposed AlgoritM}

Before presenting, the proposed algorithms, some basic concepts used in this algorithm are briefly explained. First, the prioritizing criteria of qualitative scenarios are introduced then algorithm structure including chromosome structure, creating the initial population and fitness function is presented.

\subsection{The Prioritizing Criteria Of Qualitative Scenarios In NSGa-II ALgORITHM}

In addition to prioritizing criteria of qualitative scenarios presented by Mr.Karimi [16], another criterion has added in this study to determine the amount of scenario duplication. The seven criteria are as follows:

1. Priority of Each qualitative scenario based on the shareholders' view.

2. Difficulty level to achieve or implement the scenario

3. The importance of each criterion or sub-criterion of the qualitative feature related to the scenario for the organization.

4. The amount of the impact of the scenario answer criterion on each criterion or subcriterion of the related qualitative feature

5. The amount of scenario impact on other qualitative features

6. The priority of the shareholders participating in the evaluation process

7. Not-repetitive scenarios: when prioritizing and choosing qualitative scenarios, we should be careful not to choose and prioritize repetitive scenarios. Considering this criteria will increase the selection and prioritizing accuracy.

\subsection{NSGA-II ALgORITHM STRUCTURE}

To prioritize qualitative scenarios at the right time, the meta-heuristic algorithm NSGA - II is used in this article. This algorithm is used because it is a population algorithm and is compatible with multi-objective problems, it functions systematically when dealing with non-dominated answers of each generations and the good dispersion of answers in Pareto optimal frontier. In other words, instead of fitness function, the concept of domination of answers is used in this algorithm. Chromosome structure, creating the initial population and fitness function are introduced in this part.

\subsubsection{Chromosome STRUCTURE}

In this case, the chromosome length is fixed and equal to the number of qualitative scenarios. Each scenario is as a gene in chromosome and the arrangement of these genes represents the considered prioritizing for them throughout the algorithm implementation. The purpose of this 
algorithm is to find the optimal arrangement of the scenarios. In Figure 1 the cell array structure related to each scenario is shown. The first level of this structure includes the identifiers related to scenarios' prioritizing criteria and a QID (Quality Attributes Identifier)component as the related qualitative characteristic identifier. In this figure DR(DuplicateRate) criterion is added as the seventh criterion in addition to the existing criteria of the previous research. This seventh criterion determines the repetition rate of scenarios.

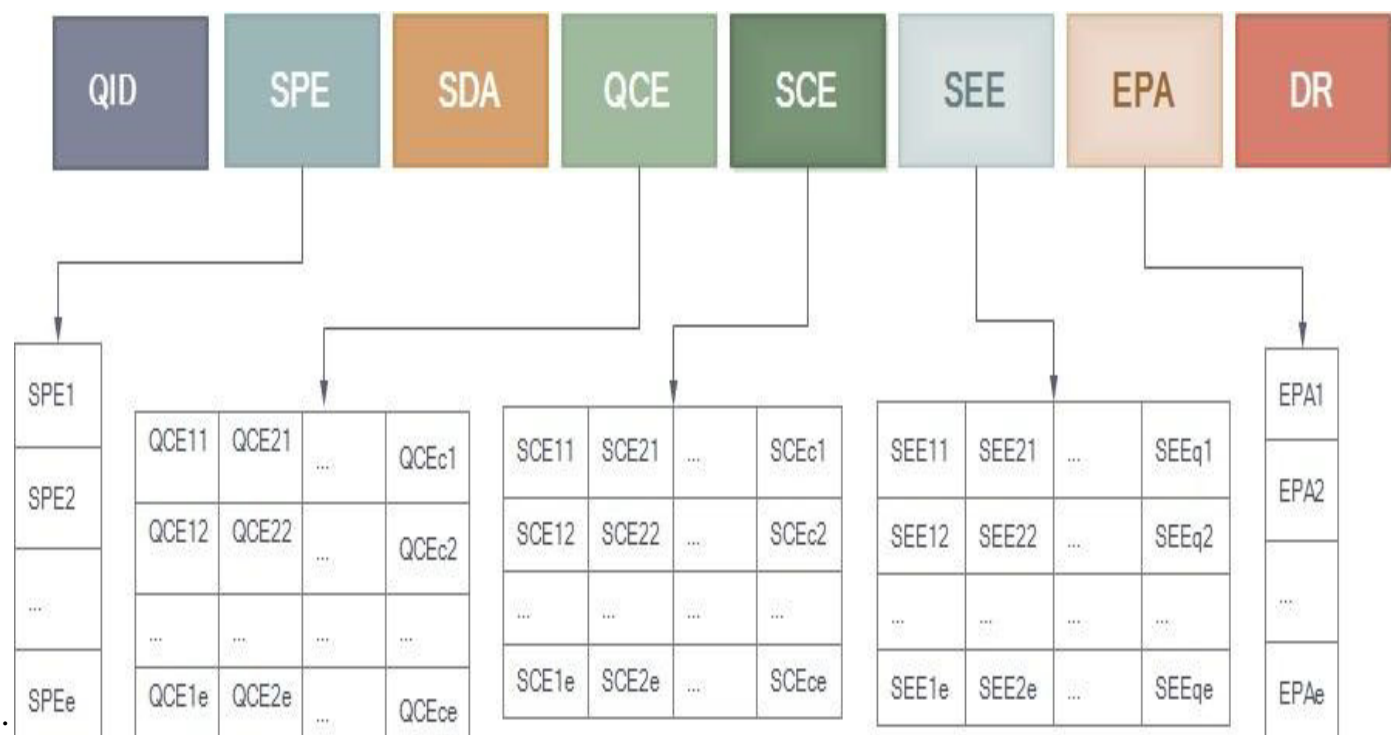

Figure 1. The structure of scenario [16] which extended by DR criterion

The variables with abbreviated names are used to show the quantitative amount in this structure. E (experts or stakeholder) constant variable shows the number of stakeholders or subjective experts. C (criteria or sub criteria) constant variable determines the number of sub-criterion of qualitative features of the related scenario. Q (quality attributes) constant variable expresses the number of all the desired qualitative features of the organization. SPE (Scenario Priority Experts) component that is an array of e number reflects the priority of scenarios based on the views of shareholders. SDA (Scenario Difficulty Access) component equals the numerical equivalent of the implementation and achievement difficulty of the scenario. QCE (Quality Criteria Experts) components is a matrix with $\mathrm{e} * \mathrm{c}$ dimensions that represents the views of stakeholders on the importance of each criterion or sub-criterion of related qualitative characteristics. SCE (Scenario Criteria Experts) component is also a matrix with $\mathrm{e}^{*} \mathrm{c}$ dimensions and determines the impact of the amount of scenarios response criteria per any criteria or sub-criteria of the relevant qualitative characteristics. SEE (Scenario Effect Experts) component is a matrix with dimensions of e * $\mathrm{q}$, which represents the positive or negative impact of this scenario on the other desired qualitative features of the organization. EPA (Experts Priority Assessment) component is an e number array determining the priority of participants in the evaluation of the architecture or organization stakeholders. This component is the same for all scenarios and there is no need to be repeated in all of them[17]. The added component in this paper is DR(Duplicate Rate)that shows the repetition rate of scenario and the more frequent scenario in the chromosome the closer the value will be to zero.

\subsubsection{Creating The Initial Population}

The first step of the algorithm is to generate the initial population. The initial population is a descendant of the original chromosomes manufactured randomly. In this problem an initial 
population of $\mathrm{N}$ chromosomes is produced; $\mathrm{N}$ is the population size or number of members of the first generation.

The best answer is related to the chromosome that the arrangement of scenarios in it includes implementing the best state in the prioritizing criteria as follows [16]:

1. Descending order of scenarios prioritizing criteria based on the organization shareholders' view.

2. Descending order of the amount of the achieving difficulty of the scenario

3. Descending order of the criteria's importance criteria or sub-criteria of qualitative characteristics

4. Descending order of the impact of the amount of scenarios response criteria per any criteria or sub-criteria of the relevant qualitative characteristics

5. Ascending order of the amount of scenarios negative impact criterion on other qualitative features.

6. The lack of duplicative scenarios in each chromosome.

\subsubsection{FITNESS FUNCTION}

This function is a criterion to determine the more appropriate or qualified answer comparing to other answers. Given that each chromosome represents a response, suitability or fitness of each chromosome in comparison with other chromosomes in the same generation can be determined by assigning a numerical value [19]. In this article, fitness function in the [17] reference is used with the difference that this function is converted into multi-objective one and $\mathbf{D R}$ (Duplicate Rate) criterion is added to it to show the repetitive rate of scenario. F (X) Fitness function for $X$ chromosome is shown by the following equation (taken from reference [17] by adding DR).

$$
F(X, S)=\sum_{i=1}^{n}\left(\left(\left(W_{1} \times F P_{i}\right)+\left(W_{2} \times F D_{i}\right)+\left(W_{3} \times F C_{i}\right)+\left(W_{4} \times F E_{i}\right)\right)^{\frac{1}{i}}\right) \times D R
$$

This function input is the one-dimensional array of $\mathrm{X}$ and cell array of $\mathrm{S}$. $\mathrm{X}$ is an array by the length of $n$ which elements show the identifiers of the scenarios existing in it. $n$ is the total number of scenarios. S cell array includes all numerical structures related to scenarios. $\mathrm{X}$ array elements show the number of scenario structure existing in $\mathrm{S}$ scenarios to calculate the fitness. The output of this function represents the amount of the input chromosome merit; the greater the value of the function, the more qualified the chromosome.

The proposed fitness function is composed of a few sub- functions, each of which is responsible for the calculation of the quantities of each prioritizing criteria of each scenario. More explanation about these sub-functions has been provided in [17] reference.

The DR Added sub-function in this article equals a value between 0 and 1; The more repetitive scenarios in the problem, the value will be closer to zero. Without any repetitive scenario in the answer or chromosome, the value equals 1 . In factDR criterion is the repetition rate at which the repetitions on each chromosome reduces a fraction of fitness function and quality. The formula for calculating DR is given in equation 2.

$$
D R=1-\frac{\text { Sum Of Duplicate Scenarios }}{\text { Number Of Scenario }}
$$

Numerator is the total number of repetitive scenarios and denominator shows scenarios the total number of scenarios. For example, if we have 4 scenarios 1, 2, 3 and 4, and a sample of the 
population as Figure 2, the total number of scenarios equals 4 and the number of repetitive scenario equals 2 and DR 2 is equal to 0.5. This means that fitness function is divided to two halves.

\begin{tabular}{|l|l|l|l|}
\hline 2 & 4 & 4 & 2 \\
\hline
\end{tabular}

Figure 2.Sample of the population

To calculate the fitness function a power $(1 / \mathrm{i})$ is also used. The reason is that the amount calculated for each scenario has to be exponentiated by its reversed rank so that in the production of new chromosomes, preferred scenarios that have a higher value place at the beginning of the chromosome.

Finally change the problem into a multi-objective one, the fitness function was defined as follows:

$$
Z 1=\sum_{i=1}^{n}\left(\left(\left(W_{1} \times F P_{i}\right)+\left(W_{2} \times F D_{i}\right)\right)^{\frac{1}{i}}\right) \times D R, Z 2=\sum_{i=1}^{n}\left(\left(\left(W_{3} \times F C_{i}\right)+\left(W_{4} \times F E_{i}\right)\right)^{\frac{1}{i}}\right) \times D R
$$

$\mathrm{Z} 1$ and $\mathrm{Z} 2$ are the target functionsin the above equation.

\section{Clarification Of NSGa-II Proposed Algorithm}

Several outputs exist per the entire output of the fitness function or output the very objectives of the problem. These outputs are important criteria in dominance and crowding distance. The proposed method flowchart is shown in Figure 3. In this flowchart first, the population parameters are determined such as mutation rate, composition, number of population samples, species size (equal to the number of scenarios), and number of frequencies. After determining the initial parameters of the problem, we create an initial population of scenarios which varieties are as the array with one row and have components equal to the number of scenarios. After creating the initial population, we improve it by a repetitive process. The improvement process of the population is in a way that per sample population, fitness function is applied on it. Fitness function output are several parameters that each of which will be compared to output parameters of fitness function of other species. If all the parameters of one species or chromosomes is more than the other species' parameters, this species dominates the compared species, and indeed is more suitable then the other. After determining the amount of species domination and recessiveness, distance navigation of all these species are calculated in all aspects or the very fitness function outputs. The process of calculating the distance navigation is similar to domination, in which any species is compared to other species. In the next stage mutation and composition operations are done on the species and the two obtained sets of answers is added to the population. After that species are arranged in terms of domination first and then in terms of distance navigation. After arranging, nPop (n Population) primary species are entered into new populations and other species are removed. These steps will be repeated until the maximum frequency is reached. 
International Journal of Computer Science \& Information Technology (IJCSIT) Vol 8, No 6, December 2016

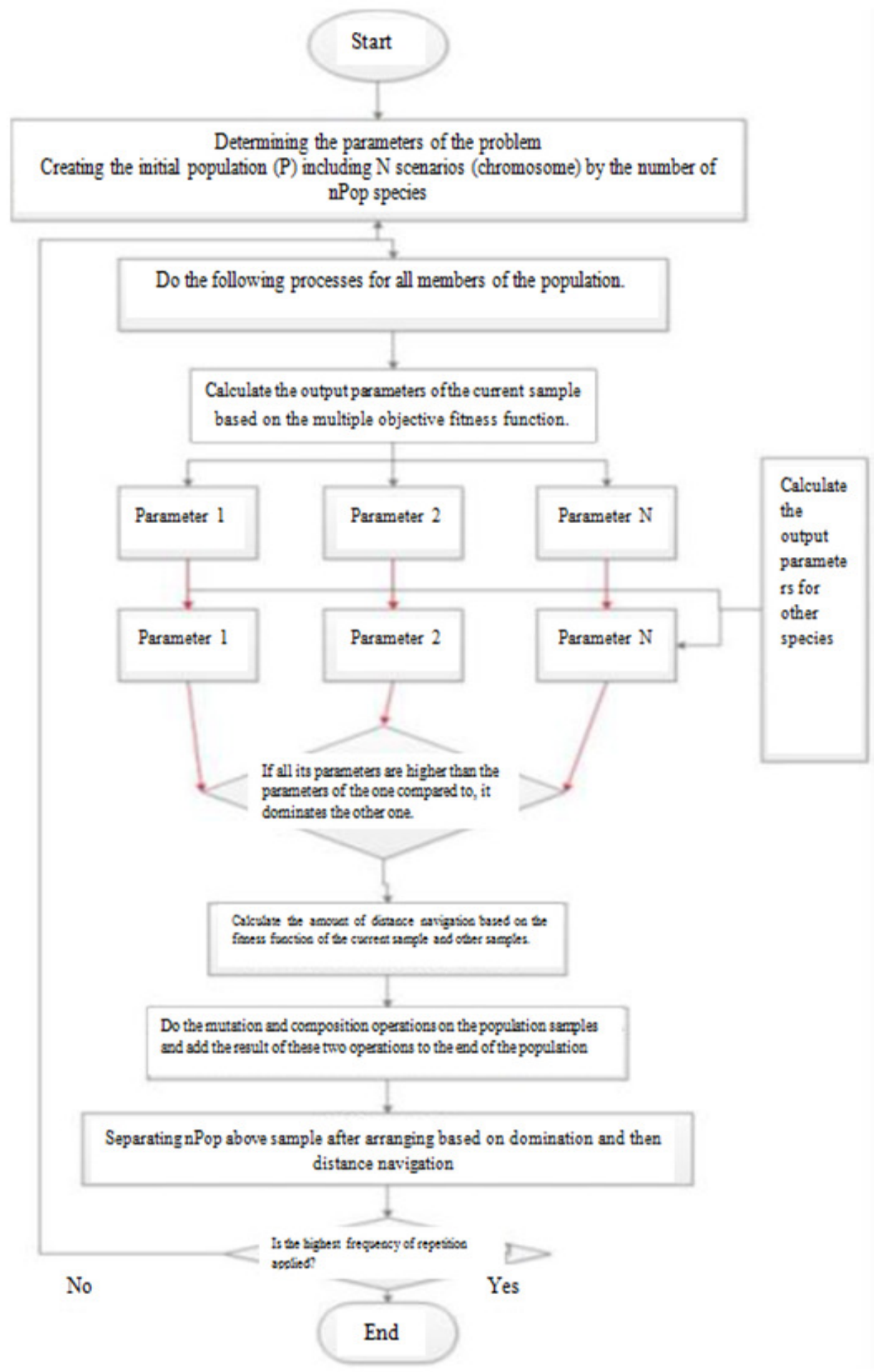

Figure 3. General Flowchart of the proposed method 


\section{Evaluation Of Proposed Algorithm}

The proposed algorithm was examined in two case studies. In the first evaluation by carrying out a case study in the field of enterprise architecture with four scenarios, the accuracy of the algorithm was investigated. The assessment criterion was that

1.the prioritizing results should be similar to the state of the one previously conducted by other methods which results were proven to be accurate,

2. Comparing and evaluating the efficiency of this method with other similar methods.

The resultsof experiments in first case study showed that scenarios priority is consistent with the results of reference [2]. In figure 4,the result of Matlabdeveloped program based on proposed algorithm for the first case study is shown. It shows that the priority of scenarios is 4,1,2 and 3 respectively. This result is the same as the report of reference [2] that confirms the accuracy of proposed method..

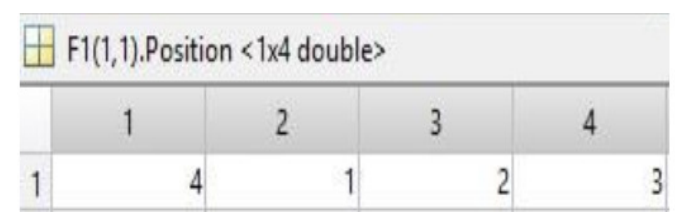

Figure 4. Result of proposed algorithm on first case study provided by Matlab program

The second case study is related to Chapter XI of reference [4] which consider 29 scenarios. The purpose of the second evaluation is to demonstrate the capabilities of applying the proposed algorithm in the field of software architecture. Therefore, by carrying out a case study in the field of software architecture, the amount of achieving this goal and its performance accuracy were analyzed. Comparison of the result of genetic algorithm presented in Figure 5 and the result of our experiment which shown in Figure 6 made it clear that our NSGA-II based algorithm removed the repetitive $16,9,8,4,19$ scenarios.

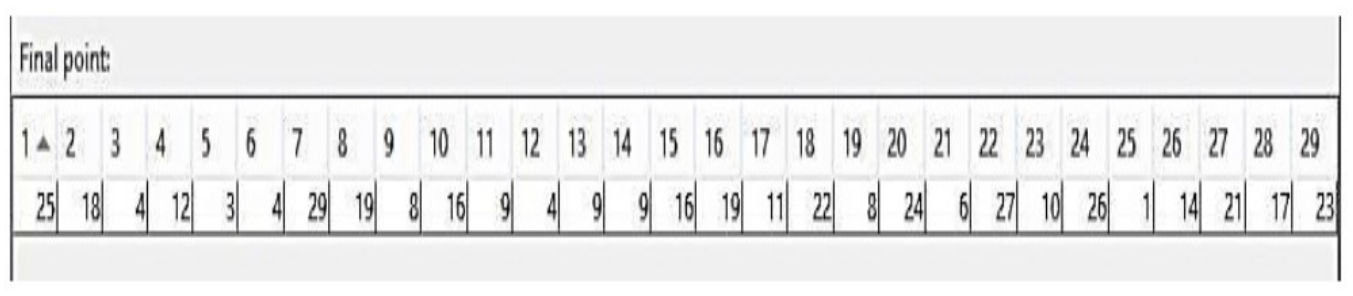

Figure 5. Result of scenarios prioritization based on previous genetic algorithm

\section{F1(1,1).Position <1×30 double>}

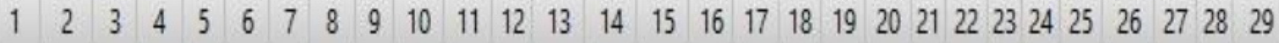

$\begin{array}{lllllllllllllllllllllllllllllll}1 & 29 & 15 & 17 & 16 & 25 & 22 & 26 & 27 & 19 & 24 & 20 & 23 & 21 & 28 & 18 & 7 & 14 & 13 & 12 & 4 & 3 & 11 & 9 & 5 & 2 & 10 & 1 & 6 & 8\end{array}$

Figure 6. Result of scenarios prioritization based on new NSGA-II algorithm 
The evaluation results which are compared with Karimi work are presented in tables 1 and 2. This results shows 44.8 and 33.053 percent improvement in comparison to Karimi genetic algorithm in first and second case studies, respectively.

Table 1. Comparison of algorithm running time compared withKarimi (first case study).

\begin{tabular}{|l|l|l|}
\hline \multirow{2}{*}{ execution time } & proposed method & Karimi method \\
\hline Percent of improvement & $4.196357(\mathrm{~s})$ & $7.610441(\mathrm{~s})$ \\
\hline
\end{tabular}

Table 2. Comparison ofalgorithm running time compared with Karimi (second case study).

\begin{tabular}{|l|l|l|}
\hline & proposed method & Karimi method \\
\hline execution time & $106.223219(\mathrm{~s})$ & $158.669815(\mathrm{~s})$ \\
\hline Percent of improvement & \multicolumn{2}{|c|}{$33.053 \%$} \\
\hline
\end{tabular}

\section{Conclusion And Future Work}

Some of the methods that existed for enterprise architecture evaluation were investigated briefly in this article. As mentioned the qualitative scenarios are used to determine the qualitative features of enterprise architecture, so more attention should be paid to the prioritizing of the scenarios in evaluating methods. Since ATAM method considered prioritization of scenarios, proposed method were developed based on ATAM. In the present study to reduce running time a new method of prioritizing scenarios based on NSGA-II algorithm was introduced. This algorithm had more detailed criteria for prioritizing qualitative scenarios in the evaluation process. The proposed algorithm has been evaluated in two stages. The results were compared to the results of Mr Karimi, who has used the genetic algorithm. The new method showed a better running time comparing to genetic algorithm. In order to obtain scenario optimal order, the proposed method prevented the repetition of the scenarios and removed the previous method flaw. As future work, researchers can consider new criteria for prioritizing qualitative scenarios and also they can implement other metaheuristicalgorithm to prioritize qualitative scenarios in a new way and then it can be compared with current work result to gain more improvement.

\section{REFERENCES}

[1] R. Khayami. "Qualitative characteristics of enterprise architecture". In Proc. Computer Science 3 World Conference on Information Technology,2011, pp. 1277-1282.

[2] M. RazaviDavoudi. "Representing a New Framework for Analyzing Qualitative Characteristics of Enterprise Architecture", PhD thesis, Faculty of Computer Engineering, Islamic Azad University, Science and Research Branch, Tehran, Iran, 2010.

[3] I. F. E. A. Developments. (January 2010). Institute for Enterprise Architecture Developments.[online] Available: http://www.enterprisearchitecture.info/ifead\%20about.htm

[4] L.Bass, P. Clements and R. Kazman, Software Architecture in Practice. 3nd ed, Addison-Wesley Professional,2012.

[5] R. Khayami. "Evaluating and Analyzing Enterprise Architecture", PhD thesis, Faculty of Engineering, Shiraz University, Shiraz, Iran, 2009.

[6] M. Razavi, F. Shams Aliee, and Amir E. SarabadaniTafresh, "A FuzzyAHP Based Approach Towards Enterprise Architecture Evaluation",InProc. 3rd European Conference on Information Management andEvaluation, Sweden, 2009, pp. 408-422.

[7] J. Schekkerman, "Enterprise Architecture Score Card Version 2.1,", Institute For Enterprise Architecture Developments, The Netherlands. 2004 
[8] OMB, OMB Enterprise Architecture Assessment Framework Version 1.5, Office of Management and Budget, USA. 2005.

[9] P. Johnson,E. Johansson, T. Sommestad, andJ. Ullberg, "A Tool for Enterprise Architecture Analysis", In Proce. 11th IEEE Enterprise Distributed Object Computing Conference, USA,2007, pp. 142-156.

[10] P. Narman, P. Johnson, R. LagerströmandM. Simonsson, "Enterprise architecture analysis with extended influence diagrams", Information Systems Frontiers. pp. 163-180, 2007.

[11] P. Johnson,R.Lagerstrom, P. Narman and M. Simonsson,"Extended Influence Diagrams for Enterprise Architecture Analysis",In Proc. 10th IEEE Enterprise Distributed Object Computing Conference,2006,pp 3-12.

[12] E. Mathias,P.Narman and M. Gammelgard , "Architecture Scenario Analysis- Estimating the Credibility of the Results," In Proc. 17th International Symposium of the Council on Systems Engineering. 2007

[13] M. Simonsson, A. Lindström, P. Johnson, L. Nordström, J. Grundbäck and O. Wijnbladh, "Scenariobased Evaluation of Enterprise - a Top-Down Approach for Chief Information Officer Decision Making", In Proc. 7th International Conference on Enterprise Information Systems (ICEIS2005), Miami, USA, May 25-28,2005, pp. 130-137.

[14] P. Närman, P. JohnsonandL. Nordström, "Enterprise Architecture: A Framework Supporting System Quality Analysis", In Proc. 11th IEEE Enterprise Distributed Object Computing Conference, 2007, pp. 130-141.

[15] M. Razavi and K. Sheikhvand, "An Ppproach towards Enterprise Architecture Analysis using AHP and Fuzzy AHP ", International Jornal of Machin Learning and Computing(IJMLC), Vol. 2,No.1, pp. 46-51, February 2012.

[16] M. Karimi, "Prioritizing Quality Scenarios in Evaluating Enterprise Architecture Using Genetic Algorithms", MS Thesis, Faculty of Computer Engineering, Islamic Azad University, Najaf Abad Branch, Isfahan, Iran, 2013.

[17] M. Karimi, Sayed M. Sharafi and M. NaderiDehkordi, "A New Approach Based on Genetic Algorithm for Prioritizing Quality Scenarios in Enterprise Architecture Evaluation", International Journal of Computer Science Engineering (IJCSE), Vol. 3 No.01, pp. 21-31, 2014.

[18] J. Klein, M. Gagliardi and J. Michael, "A Workshop on Analysis and Evaluation of Enterprise Architectures", Software Engineering Institute, pp 429, 2010.

[19] M. SH. Javi, (2011), Implementing and Solving Applied Issues by Genetic Algorithm, Arya Pazhouh Publications.

\section{AUTHORS}

MarziehEskandari(m.eskandari066@gmail.com) was born in March, 1987 in Shahrekord, Iran. She defended her master thesis, "Prioritizing Quality Scenarios in Enterprise Architecture Evaluation Using NSGA-II Algorithm”, in January, 2016. She was graduatedwith MSc degree in software engineering at Islamic Azad University, Najafabad Branch, Najafabad, Iran.Her research interests are software engineering, enterprise architecture, and software architecture.

Akbar Nabiollahi-Najafabadi(a.nabi@pco.iaun.ac.ir, nabi.akbar@gmail.com) was born in 1970 in Najafabad, Iran. He received his BSc in software engineering from Isfahan University of Technology in 1994. Continuing education, he received his MSc in software engineering from Islamic Azad University, Najafabad Branch in 2002. He conducted his doctoral studies in computing science, with the orientation of Information technology, in UniversitiTeknologi Malaysia (UTM) from 2008 to 2012. His dissertation was about "A Proposed Integrated Framework for Enterprise Architecture and Information Technology Service Management". His work experience consists of various projects in software engineering, IT service management, and information systems. Since 2012, he has been the assistant professor of the Faculty of Computer Engineering in Islamic Azad University, Najafabad Branch, Iran. His publications include many journal and conference papers. His research interests are enterprise architecture, information systems, and IT service management. 